\title{
Expression of cell cycle regulatory proteins in endometrial adenocarcinoma: variations in conventional tumor areas and in microcystic, elongated and fragmented glands
}

\author{
Colin JR Stewart ${ }^{1,3}$, Maxine L Crook$^{1}$, Yee C Leung ${ }^{2}$ and Michael Platten ${ }^{1}$ \\ ${ }^{1}$ Department of Histopathology, King Edward Memorial Hospital, Perth, Western Australia, Australia; \\ ${ }^{2}$ Department of Gynecologic Oncology, King Edward Memorial Hospital, Perth, Western Australia, Australia \\ and ${ }^{3}$ School of Pathology and Laboratory Medicine, University of Western Australia, Western Australia, \\ Australia
}

\begin{abstract}
Endometrial adenocarcinomas may show a distinctive pattern of invasion characterized by the presence of microcystic, elongated and fragmented glands, often most evident along the advancing tumor margin. Earlier, we have shown that these changes appear restricted to low-grade endometrioid carcinomas, many of which show focal mucinous differentiation and lymphovascular space invasion. However, the molecular alterations associated with this morphological alteration are not known. In this study, we have examined immunoreactivity for the cell cycle regulatory proteins cyclin D1, p16 and $\beta$-catenin in 22 endometrial carcinomas, specifically comparing the results in conventional tumor areas and in foci in which the glands exhibited microcystic, elongated and fragmented appearances. The conventional neoplastic glands exhibited cyclin D1 and p16 expression in most cases, with $>\mathbf{5 0} \%$ tumor cells positive in $\mathbf{8}$ cases and 11 tumors, respectively. Membranous expression of $\beta$-catenin was usually preserved, with variable cytoplasmic and nuclear staining. Cyclin D1 and $\beta$-catenin predominantly stained cells at the peripheral or basal aspect of the conventional glands, whereas p16 was more uniformly expressed centrally. Tumor foci composed of microcystic, fragmented and elongated glands showed strong expression of cyclin D1 and p16, sometimes in contrast to unstained contiguous or adjacent conventional neoplastic elements, and there was also loss or fragmentation of membranous $\beta$-catenin staining. Intravascular tumor cells also expressed cyclin D1 and p16 and therefore the immunostains often highlighted subtle foci of lymphovascular invasion. The heterogenous expression of cell cycle regulatory proteins within endometrial adenocarcinoma illustrates the importance of assessing microanatomical variations in immunoreactivity, particularly at the advancing margin of tumors. The upregulation of cyclin D1 and $\mathrm{p16}$, together with loss of membranous $\beta$-catenin expression in microcystic, fragmented and elongated glands, is similar to epithelial-mesenchymal transitions observed in other malignancies and suggests that this pattern of invasion represents an active rather than a degenerative cellular process.
\end{abstract}

Modern Pathology (2009) 22, 725-733; doi:10.1038/modpathol.2009.33; published online 6 March 2009

Keywords: endometrial; carcinoma; invasion; MELF; epithelial-mesenchymal transition; immunohistochemistry

Endometrial carcinomas can be divided into two major groups on the basis of clinicopathological, immunohistological and molecular features., ${ }^{1,2}$ The more common (type 1) subgroup accounts for $80-85 \%$ of cases and mainly comprises endometrioid adenocarcinomas of low-to-intermediate grade. These tumors typically occur in perimeno-

Correspondence: Dr CJR Stewart, Department of Histopathology, King Edward Memorial Hospital, Subiaco, 6008 Perth, Western Australia, Australia.

E-mail: colin.stewart@health.wa.gov.au

Received 11 December 2008; revised 15 January 2009; accepted 16 January 2009; published online 6 March 2009 pausal and younger postmenopausal patients, often arise in hyperestrogenic states and are associated with atypical endometrial hyperplasia/endometrial intraepithelial neoplasia. Frequently, type 1 carcinomas are confined to the uterine corpus at the time of diagnosis (stage 1), and the prognosis in such patients is generally favorable with an overall 5-year survival of $85-95 \%$. In contrast, type 2 endometrial carcinomas often occur in older patients, are not associated with estrogenic stimulation, show non-endometrioid and high-grade histological appearances, and have a poorer prognosis. ${ }^{1,2}$

The pathogenesis of uterine endometrioid adenocarcinoma has been subject to intense investigation 
in recent years, and several common molecular abnormalities have been identified, including inactivation of the tumor suppressor gene PTEN, mutations of the $k$-ras oncogene and microsatellite instability. $^{3-6}$ Additional changes that occur in a significant proportion of cases include dysregulation of the Wnt and retinoblastoma $(\mathrm{Rb})$ signaling pathways. These are complex and interconnected pathways, each of which includes multiple positive and negative regulatory factors that influence cellular proliferation and differentiation. ${ }^{7-11}$ Activation of the Wnt pathway leads to decreased degradation, and thus increased cytoplasmic accumulation, of $\beta$-catenin. The subsequent nuclear translocation of $\beta$-catenin induces cell proliferation, mainly through the upregulation of factors such as c-myc and cyclin D1. $\beta$-Catenin also plays an important role in maintaining the adhesion and polarity of epithelial cells through its association with E-cadherin on the cell membrane. Reduced expression of the E-cadherin/ $\beta$-catenin complex is considered a fundamental step in the progression of many types of carcinoma, notably as a cardinal feature of epithelial-mesenchymal transition, which permits epithelial cells to acquire motility and display invasive properties. ${ }^{12-14}$

The Rb pathway exerts control mainly on cell proliferation by regulating cell progression through the G1/S phases of the cell cycle. The Rb protein inhibits cell division and therefore its inactivation by complexes of cyclin-dependent kinases (cdks) and cyclins, such as cdk4/6 and cyclin D1, has a pro-proliferative effect. In turn, the cdk-cyclin complexes are negatively regulated by cdk inhibitors, such as CDK4INK (also known as p16 ${ }^{\text {INK4a }}$ and hereafter referred to as p16). Thus, p16 protein normally exerts an antiproliferative or, in the neoplastic context, a tumor suppressor effect. The $\mathrm{Rb}$ pathway may be disrupted at multiple levels, including inactivating mutations or deletions of the $R b$ gene itself, overexpression of cyclin D1 or cdk, and downregulation of p16, and such abnormalities alone or in combination have been identified in many neoplasms, including endometrial carcinomas. ${ }^{15}$ Although the Wnt and Rb pathways have independent activities, they also have overlapping functions, notably through the regulation of cyclin $\mathrm{D} 1$, the activity of which is promoted by $\beta$-catenin but inhibited by p16. Recent studies also suggest that nuclear $\beta$-catenin may exert direct effects on p16, which are linked to squamous (morular type) differentiation in endometrioid adenocarcinoma. ${ }^{16}$

In endometrial cancer, abnormalities of the Wnt and $\mathrm{Rb}$ pathways have been studied at the genotypic level and also with immunohistochemical techniques that evaluate alterations in protein expression. However, although some of the latter studies have taken into account both the intensity and the extent of immunoreactivity, few have considered the specific distribution of protein expression at the microanatomical level. It is becoming clear that tumors are heterogenous with regard to molecular and immunophenotypical features and therefore an 'overall' estimate of protein expression may obscure changes in specific areas, such as the advancing tumor margin in which processes, such as epithelial-mesenchymal transition, are most active. ${ }^{11,17}$ We have noted earlier that endometrial carcinomas exhibiting a distinctive pattern of invasion comprising microcystic, elongated and fragmented ('MELF') glands ${ }^{18}$ show specific pathological associations and morphological changes, some of which are suggestive of epithelial-mesenchymal transition. ${ }^{19,20}$ Therefore, in this study, we have further explored the cellular alterations at the invasive margin of endometrial adenocarcinoma by comparing the expression of the cell-cycle-related proteins, cyclin $\mathrm{D} 1, \beta$-catenin and $\mathrm{p} 16$, between conventional tumor areas and foci exhibiting MELF pattern invasion.

\section{Materials and methods}

Twenty-two endometrial cancers exhibiting a MELF type of myometrial invasion were identified within the histopathology files of King Edward Memorial Hospital, Perth, Western Australia. Nineteen of these cases were selected from 27 endometrial carcinomas showing MELF features accessioned in our institution between July 2005 and June $2008,{ }^{19}$ and there were three subsequently identified tumors. All MELF-positive cases were low-grade (FIGO grade 1 or 2) endometrioid adenocarcinomas although three cases had separate, discreet areas of high-grade (de-differentiated) carcinoma. ${ }^{21}$ The MELF pattern changes were as earlier described comprising dilated (microcystic), elongated and fragmented glands, usually most evident at the deepest myoinvasive aspect of the tumors. ${ }^{18,19}$ The cases selected for study exhibited multiple MELF-type foci in at least one tumor block that was used for the immunohistochemical evaluation.

Clinicopathological data, including patient age, tumor grade and stage (FIGO), and the presence of lymphovascular space invasion were obtained from the histopathology reports and from review of the tumor slides.

\section{Immunohistochemistry}

The tumors were stained with the panel of antisera outlined in Table 1 . The staining was performed on serial $4-\mu \mathrm{m}$ sections to permit the correlation of the different immunohistochemical markers in specific tumor areas. The detection kit was the LSAB 2 HRP system (K0675; Dako, NSW, Australia) with liquid diaminobenzidine and substrate chromogen system (K3468; Dako). The results were compared with those of the corresponding hematoxylin-and-eosinand cytokeratin (CK)7-stained sections. We have noted earlier that CK7 is strongly expressed in areas of MELF pattern invasion within endometrial 
Table 1 Summary of antisera and immunohistochemical findings in conventional and MELF pattern tumor components

\begin{tabular}{|c|c|c|c|}
\hline Antibody & Source; dilution & Conventional tumor areas & MELF tumor areas \\
\hline Cyclin D1 & $\begin{array}{l}\text { Dako, NSW, } \\
\text { Australia; 1:100 }\end{array}$ & $\begin{array}{l}\text { Variable expression in } 20 \text { tumors ( }>50 \% \text { cells } \\
\text { positive in eight cases). Peripheral/basal } \\
\text { glands more consistently stained. }\end{array}$ & $\begin{array}{l}\text { Strongly positive in } 20 \text { cases, sometimes in contrast } \\
\text { to unstained adjacent or contiguous conventional } \\
\text { tumor areas. }\end{array}$ \\
\hline$\beta$-Catenin & $\begin{array}{l}\text { Novocastra, } \\
\text { Newcastle, UK; } \\
1: 4000\end{array}$ & $\begin{array}{l}\text { Membrane labeling in } 18 \text { tumors, often with } \\
\text { cytoplasmic staining in peripheral/basal } \\
\text { glands. Focal nuclear staining in six cases. } \\
\text { Squamous morules positive. }\end{array}$ & $\begin{array}{l}\text { Complete loss of membrane staining in most areas, } \\
\text { usually with weak cytoplasmic reactivity. Focal } \\
\text { nuclear staining in two cases. }\end{array}$ \\
\hline p16 & $\begin{array}{l}\text { Santa Cruz, CA, } \\
\text { USA; 1:50 }\end{array}$ & $\begin{array}{l}\text { All cases at least focally stained ( }>50 \% \text { cells } \\
\text { positive in } 11 \text { cases). Squamous morules } \\
\text { positive. Variable distribution but central } \\
\text { glands more uniformly stained. }\end{array}$ & $\begin{array}{l}\text { Moderate-to-strong immunoreactivity in } 20 \text { cases, } \\
\text { sometimes in contrast to unstained adjacent or } \\
\text { contiguous conventional tumor areas. }\end{array}$ \\
\hline
\end{tabular}

adenocarcinoma and therefore could be used as a reference for comparing the additional immunohistological preparations. ${ }^{20}$ The staining patterns were assessed separately in the predominant areas of conventional myoinvasive adenocarcinoma and at the advancing tumor margin, especially in the areas exhibiting MELF-type features. In those sections that included several MELF foci, the predominant pattern was recorded although in practice staining was fairly uniform in these areas. All staining batches included appropriate controls.

The study received institutional ethics approval.

\section{Results}

The immunohistochemical findings and the clinicopathological details of the 22 cases are summarized in Tables 1 and 2, respectively.

Cyclin D1 immunoreactivity was mainly confined to the nuclei and was observed within the conventional glandular component of 20 tumors. However, there was a wide range in the extent of immunoreactivity, and only eight cases showed expression in $>50 \%$ tumor cells. Frequently, cyclin D1 was more uniformly and strongly expressed toward the basal or peripheral aspect of the conventional neoplastic glands, with reduced or absent staining centrally (Figure 1). MELF-type foci were strongly cyclin D1 positive in most cases, sometimes in contrast to adjacent or contiguous unstained conventional-type tumor glands (Figures 2 and 3). Thus, cyclin D1 staining often highlighted areas of MELF pattern invasion, including inconspicuous small neoplastic cords of cells, single infiltrating cells and adjacent intravascular tumor nests (Figure 3). Occasional labeling of endothelial nuclei with cyclin D1 was also observed.

$\beta$-Catenin immunoreactivity was observed within the conventional tumor glands in all cases. Eighteen tumors showed a strong and complete membranous expression similar to that seen in the normal endometrial epithelium, whereas a weaker and fragmented membranous labeling was seen in four cases. Many tumors also showed an increased intensity of
Table 2 Summary of clinicopathological details in 22 MELFpositive endometrioid endometrial carcinomas

\begin{tabular}{lr}
\hline Mean age (range) & $69.9(51-94)$ \\
\hline Grade (\%) & \\
Grade 1 & $15(68.2)$ \\
Grade 2 & $4(18.2)$ \\
De-differentiated & $3(13.6)$ \\
& \\
Stage (\%) & \\
1B & $6(27.3)$ \\
1C & $9(40.9)$ \\
2 & $2(9.1)$ \\
3 & $5(22.7)$ \\
Vascular invasion (\%) & $15(68.2)$
\end{tabular}

$\beta$-catenin expression toward the basal aspect of the larger tumor glands as a result of combined membranous and cytoplasmic staining (Figure 4). In contrast, the neoplastic epithelium within the center of the glands showed a more restricted membrane labeling, especially in areas of mucinous differentiation. Nuclear $\beta$-catenin expression was identified in six tumors but showed no obvious pattern of anatomical distribution. Squamous differentiation was present in five adenocarcinomas, four of which exhibited a predominantly morular pattern, and these areas showed cytoplasmic and nuclear $\beta$-catenin expression. Immunoreactivity was generally most marked toward the basal (basement membrane) aspect of squamous areas. All MELF pattern tumor foci showed an altered $\beta$-catenin expression. In most cases, there was a complete loss of membrane labeling typically associated with weak and diffuse cytoplasmic staining (Figures 5 and 6). Nuclear $\beta$-catenin expression was observed clearly only in two cases although the interpretation of staining sometimes was difficult due to the attenuated and disrupted nature of the neoplastic epithelium in the MELF pattern areas. Intravascular tumor nests often exhibited nuclear and/or cytoplasmic $\beta$-catenin immunoreactivity and there was partial membranous labeling in the center of some larger intravascular aggregates. Cytoplasmic 

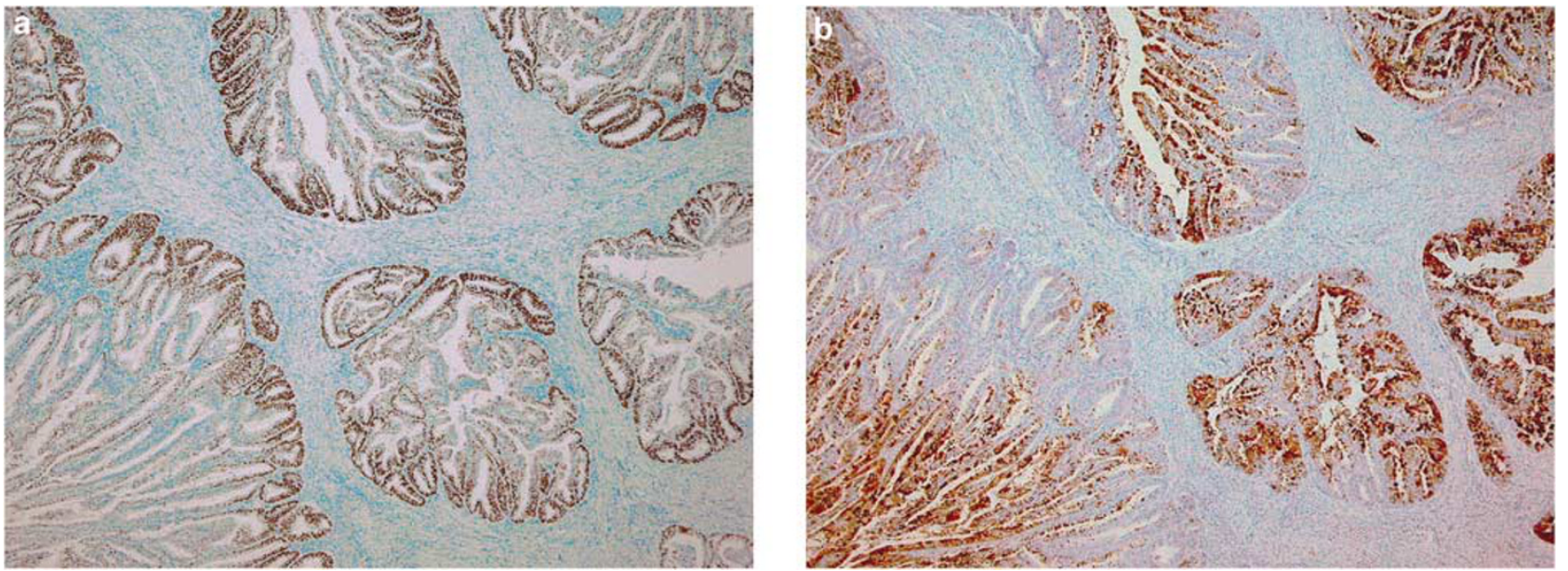

Figure 1 Immunohistochemistry for cyclin D1 (a) and p16 (b) within conventional tumor area. Cyclin D1 is most strongly expressed toward the peripheral aspect of the glands, whereas p16 staining is more marked centrally.
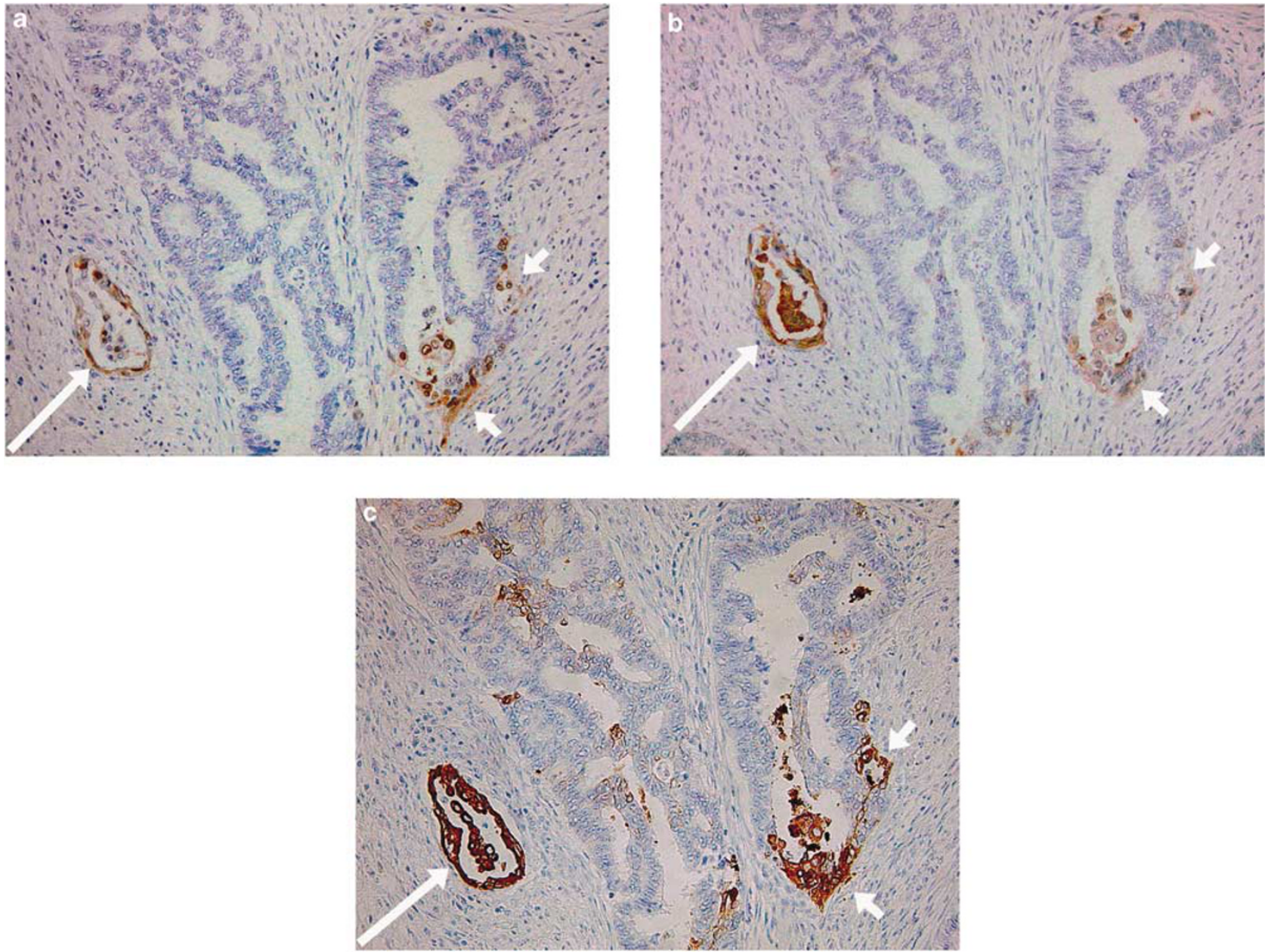

Figure 2 Immunohistochemistry for cyclin D1 (a), p16 (b) and CK7 (c). The conventional tumor glands are unstained but MELF pattern foci in continuity with the glands (arrowheads) and separately within the stroma (arrow) are positive with all antisera.

$\beta$-catenin staining was also observed focally within reactive stromal cells around MELF areas in four cases.
Immunohistochemistry for p16 showed nuclear and cytoplasmic labeling within the conventional component of all tumors, but there a was highly 

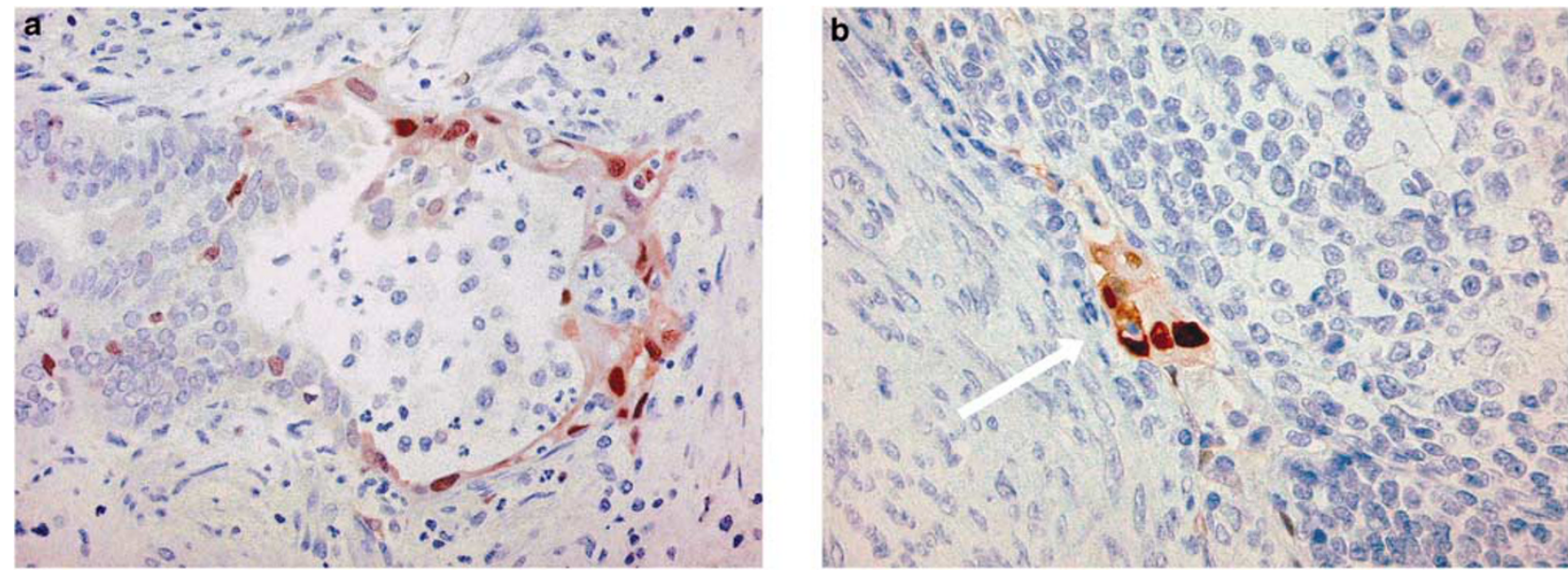

Figure 3 (a) Cyclin D1 is expressed within the MELF-type tumor area (right field), whereas the contiguous conventional tumor gland (left field) is mainly negative. (b) A small focus of intravascular tumor (arrow) is highlighted by cyclin D1 staining.
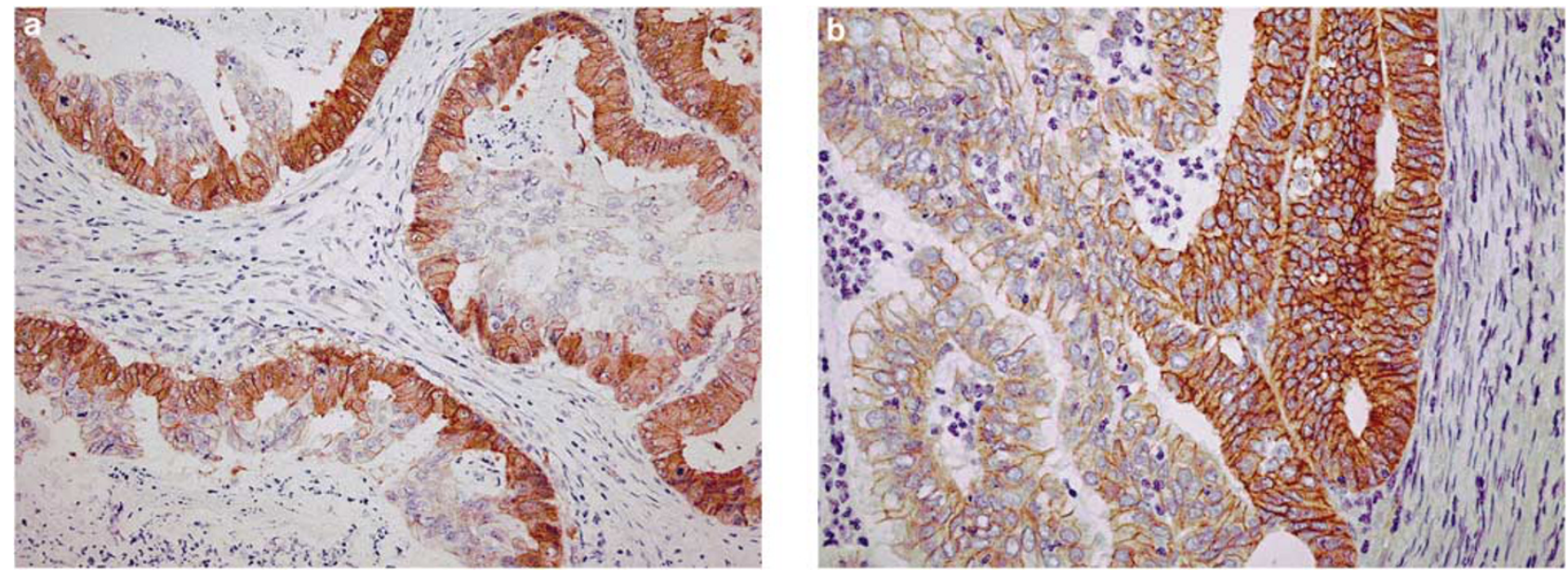

Figure 4 (a and b) Immunohistochemistry for $\beta$-catenin shows a more uniform expression toward the periphery of conventional tumor areas.
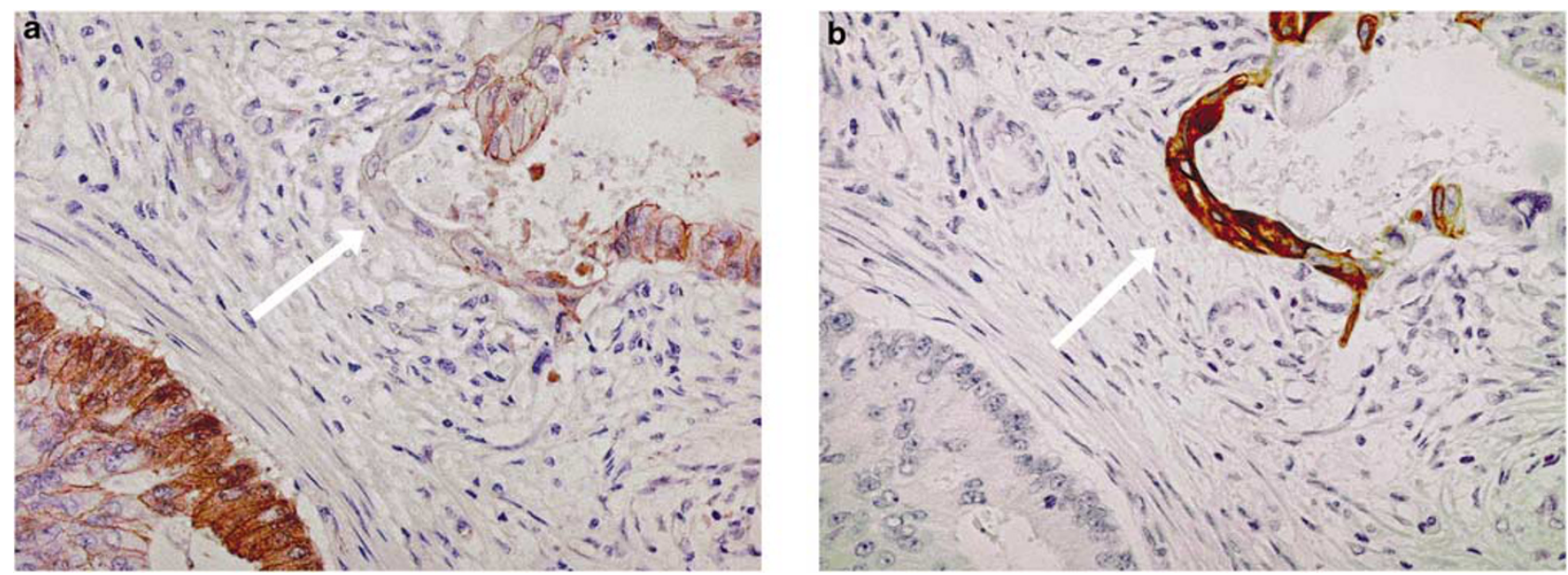

Figure 5 (a) $\beta$-Catenin expression is reduced within the MELF pattern area (arrow) compared with that in conventional tumor glands (upper right and lower left fields). (b) Note that the expression of CK7 corresponds to the MELF-type tumor alteration. 
variable expression in individual cases ranging in positivity from $<5$ to $>80 \%$. Eleven tumors showed p16 expression in $>50 \%$ of neoplastic cells. Staining was often highly localized, being restricted to small clusters of cells or even single cells within otherwise negative glands. Foci of morular-type squamous differentiation were p16 positive, and in some cases, there was a suggestion of a more uniform staining toward the center of large tumor glands (Figure 1) but often there was no clear correlation between the distribution of immunoreactivity and tumor morphology. However, MELF pattern areas of invasion were consistently p16 positive and, as with cyclin D1, the immunoreactivity sometimes contrasted with that of adjacent negative conventional-type glands (Figure 2). Tumor nests within vascular spaces also were usually p16 positive (Figure 7). In one tumor showing

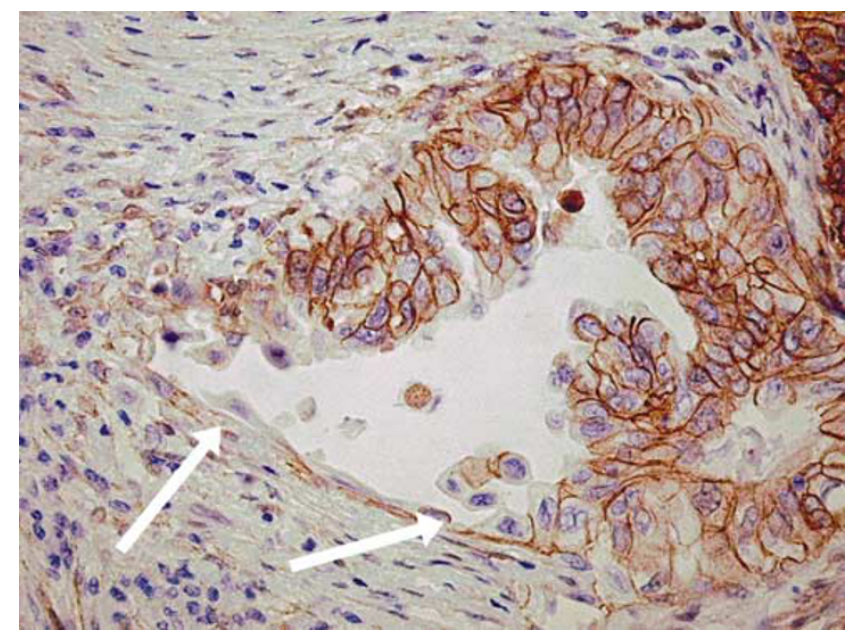

Figure 6 Immunohistochemistry showing loss or fragmentation of $\beta$-catenin expression within MELF area (arrows) compared with retained membranous staining in contiguous conventional gland (upper and right fields). de-differentiation, the high-grade component was included in the block selected for immunohistochemistry: there was diffuse and strong p16 expression within the de-differentiated tumor cells in this case.

\section{Discussion}

MELF pattern invasion in endometrial adenocarcinoma is characterized by the loss of conventional glandular architecture, attenuation of the neoplastic epithelium and infiltration of the stroma by small nests of cells and individual tumor cells often associated with a prominent fibromyxoid stromal alteration. These changes are similar to those observed in epithelial-mesenchymal transition at the advancing margin of other tumors, such as colorectal carcinoma. ${ }^{12-14,22,23}$ Earlier, we have noted that areas of MELF-type invasion are strongly positive for CK7 and show reduced E-cadherin and hormone receptor expression. ${ }^{20}$ These findings sometimes differed from those of the predominant conventional tumor areas, illustrating the importance of correlating the immunophenotypic findings with tumor distribution and morphology. In this study, we have further investigated the importance of microanatomical relationships in endometrial adenocarcinoma by comparing the expression of cell-cycle-related proteins within specific tumor areas, particularly in foci showing MELF pattern invasion.

Cyclin D1 protein is not expressed in the normal endometrium or is present only in rare cells. In contrast, immunoreactivity for cyclin D1 has been recorded in $40-69 \%$ of endometrial adenocarcinomas, ${ }^{24-29}$ and in some studies, such an overexpression has correlated with a higher grade or stage. ${ }^{24,28}$ Our findings are comparable as most tumors were at least focally positive and eight cases $(36 \%)$ showed
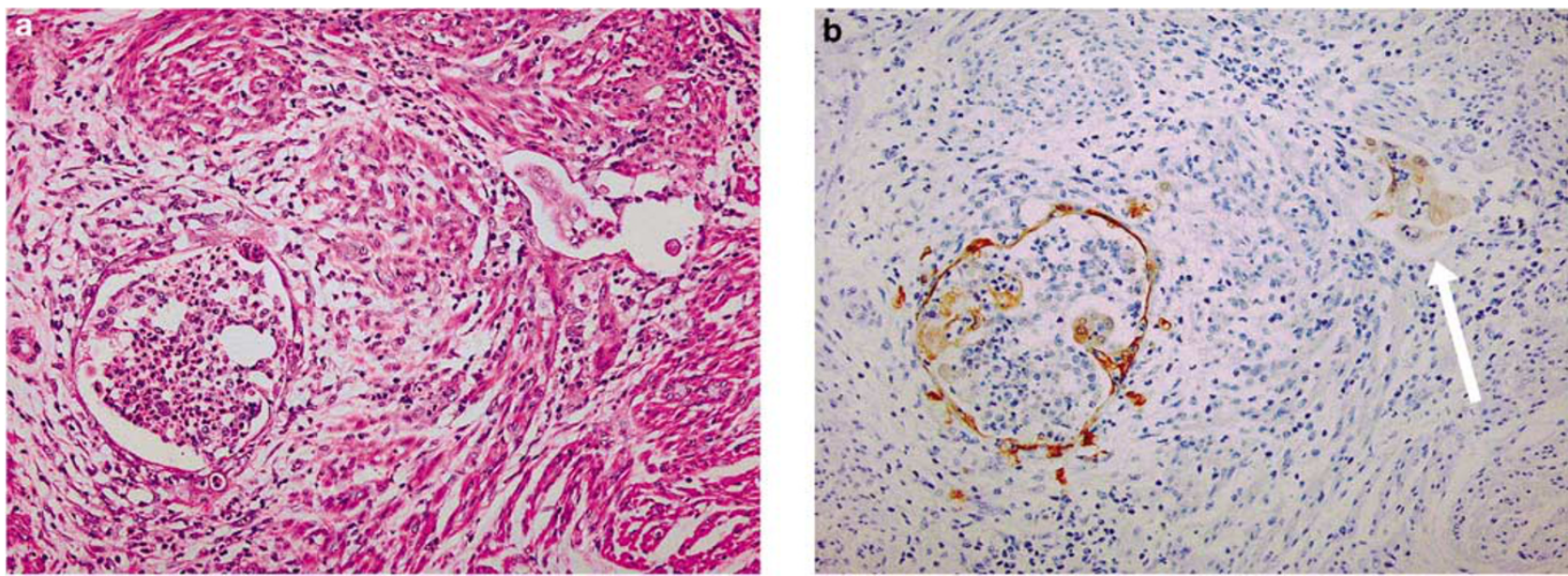

Figure 7 (a) MELF-type gland (left field) with characteristic attenuated epithelium, intralumenal neutrophils and surrounding fibromyxoid inflammatory reaction. (b) Immunohistochemistry for p16 labels the microcystic gland and also highlights an adjacent focus of intravascular invasion (arrow). 
cyclin D1 staining in $>50 \%$ cells. To our knowledge, only one earlier investigation has specifically examined the microanatomical distribution of cyclin D1 staining in endometrial carcinomas, and no difference was found between the central and the advancing aspects of these cases. ${ }^{30}$ However, in this study, the advancing tumor margin was defined by a microscopic measurement (deepest $1.2 \mathrm{~mm}$ of tumor) rather than by a morphological alteration, as seen in MELF-type invasion, and therefore, the findings are not directly comparable with those presented herein. We found that cyclin D1 expression within the conventional tumor areas was most consistent and intense around the peripheral or basal aspect of the glands. Cyclin D1 was also consistently expressed in foci of MELF pattern invasion, including isolated microcystic glands, single infiltrating tumor cells and intravascular foci. In some tumors, the localized distribution of staining was such that only MELF-type areas were positive, and therefore the tumors would have been regarded as 'negative' for cyclin D1 using the overall immunohistological scoring systems applied in some earlier studies. These findings illustrate the importance of considering microanatomical variation in staining and lend further support to the hypothesis that MELF pattern invasion represents an active rather than a degenerative process during the progression of endometrial carcinoma. ${ }^{19,20}$ Expression of cyclin D1 in MELF areas would also be consistent with epithelial-mesenchymal transition as cyclin D1 is characteristically upregulated during this process in other types of carcinoma. ${ }^{12-14,22,23}$

Abnormalities of $\beta$-catenin have also been shown in a significant proportion of endometrial neoplasms. ${ }^{3,5}$ Mutations have been recorded in $13-31 \%$ cases and appear more common in lowgrade endometrioid adenocarcinomas, ${ }^{31-36}$ whereas an abnormal immunolocalization in the form of reduced membrane staining with diffuse cytoplasmic and/or nuclear staining has been recorded in $25-76 \%$ cases. ${ }^{34-39}$ Most $\beta$-catenin mutations lead to decreased protein degradation and are associated with altered (usually nuclear) immunoreactivity. ${ }^{32,35}$ However, the converse is not always true as dysregulation of many other factors can lead to the abnormal localization of $\beta$-catenin, reflecting the multiple pathways that control the distribution and metabolism of this protein. In this study, immunoreactivity was observed within the conventional glandular component of all tumors, typically with cell membrane distribution similar to that seen in the normal endometrial epithelium. However, there was also increased staining toward the basal aspect of these glands as a result of cytoplasmic staining and, in some cases, nuclear localization. In contrast, the neoplastic elements at the advancing tumor margin and, in particular, areas exhibiting MELF pattern changes showed reduced or absent membranous $\beta$-catenin expression. Normally, $\beta$-catenin links E-cadherin to cell-adhesion complexes and cytoplasmic intermediate filaments that modulate cell motility and polarity. Thus, downregulation of membrane-bound $\beta$-catenin is consistent with the process of epithelial-mesenchymal transition. However, convincing nuclear staining was identified in only two cases in this series, a finding that differs from that observed in colorectal carcinoma in which foci of budding invasion, corresponding to epithelial-mesenchymal transition, typically show nuclear $\beta$-catenin expression. ${ }^{12,22,23}$ Therefore, alternate pathways may be more critical in upregulating cyclin D1 during the invasive process in endometrial cancers. We also noted $\beta$-catenin expression within reactive stromal cells surrounding MELF areas in some tumors. The significance of this finding is not clear, particularly as this was always a focal finding. However, the importance of tumorstromal interactions in cancer progression is increasingly recognized and it is possible that factors secreted by reactive cellular elements may help propagate tumor invasion. ${ }^{40}$

The interpretation of p16 immunoreactivity in endometrial cancer is problematic as it is not always clear whether a 'positive' or 'negative' staining reaction represents the abnormal finding. In gynecological pathology generally, p16 immunostaining has been used most widely as an adjunct to diagnosis in cervical biopsy specimens. ${ }^{41}$ Normal cervical epithelium and most reactive cervical lesions are negative or only focally positive for p16, whereas the majority of neoplastic lesions show diffuse nuclear and cytoplasmic expression. This reflects HPV-related inactivation of the Rb protein, which causes a loss of negative feedback on p16 and hence an increase in immunoreactive, but functionally ineffectual, p16 protein. ${ }^{41}$ The situation in endometrium differs in that normal proliferative endometrium expresses p16 protein and, with rare exceptions, endometrial neoplasia is not associated with HPV infection. Therefore, it can be argued that a 'positive' staining reaction for p16 protein in endometrial carcinoma represents preservation of normal expression, whereas a reduction of immunoreactivity is pathological. In accord with this interpretation, several investigators have described reduced or absent p16 expression in $6-74 \%$ of endometrial carcinomas, ${ }^{42-46}$ and in one report this corresponded to reduced levels of $p 16$ mRNA. ${ }^{46}$ The molecular basis for this downregulation is not clear as mutations, deletions and promoter methylation of the $p 16$ gene appear to be relatively infrequent. The alternative interpretation, which seems to have been adopted by other investigators, is that the expression (or 'overexpression') of p16 in endometrial carcinoma is abnormal, analogous to the situation in cervix. ${ }^{47-51}$ A very wide range in p16 immunoreactivity $(7-83 \%)$ has been reported in endometrial cancer, and this may be partly explained by differences in case selection, technical factors and varied definitions of 'positive' staining. It has been noted earlier that, when present, p16 immunoreactivity in 
endometrial carcinoma is typically focal and this may be helpful in distinguishing primary endometrial from endocervical adenocarcinomas. ${ }^{49,50}$ We also found a wide variation in p16 immunoreactivity within this series, and $11 / 22$ tumors showed staining in $>50 \%$ neoplastic cells. There was also a marked heterogeneity of staining in individual cases, and p16 expression in conventional tumor areas was often strikingly focal in distribution, sometimes limited to single cells within glands. Foci of squamous/morular differentiation were typically p16 positive as has been recorded earlier, ${ }^{16}$ and occasionally immunoreactivity was more prominent toward the central aspect of larger glands. However, often there was no clear correlation between the distribution of p16 staining and the conventional tumor pattern. In contrast, MELF pattern areas of invasion showed p16 expression consistently and, in some tumors, staining was almost restricted to these areas. These findings are in accord with those of Horree et al, ${ }^{30}$ who reported increased p16 staining at the invasive front of endometrial carcinomas, although the authors did not correlate immunoreactivity with morphological findings. The upregulation of p16 expression in association with cyclin D1 has also been described at the invasive front of colorectal carcinoma. ${ }^{52,53}$

In summary, this study has shown that the immunoreactivity of cell cycle regulatory proteins in uterine low-grade endometrioid adenocarcinomas varies according to microanatomical distribution. Cyclin D1 and $\beta$-catenin are most uniformly expressed toward the peripheral or basal aspect of conventional tumor glands. Foci of MELF pattern invasion strongly express p16 and cyclin D1, and often such immunoreactivity differs from that of adjacent or contiguous conventional glandular areas. In contrast, the epithelium within MELF pattern areas shows loss of membranous $\beta$-catenin localization. These findings indicate that the morphological changes characteristic of MELF invasion are associated with distinct and anatomically localized molecular alterations within the neoplastic epithelium, and support the hypothesis that this process represents an active cellular event during endometrial cancer invasion.

\section{Conflict of interest}

The authors have no conflict of interest to declare.

\section{References}

1 Amant F, Moerman P, Neven P, et al. Endometrial cancer. Lancet 2005;366:491-505.

2 Sorosky JI. Endometrial cancer. Obstet Gynecol 2008;111:436-447.

3 Abal M, Planaguma J, Gil-Moreno A, et al. Molecular pathology of endometrial carcinoma: transcriptional signature in endometrioid tumors. Histol Histopathol 2006;21:197-204.
4 Salvesen HB, Asklen LA. Molecular pathogenesis and prognostic factors in endometrial carcinoma. APMIS 2002;110:673-689.

5 Hecht JL, Mutter GL. Molecular and pathologic aspects of endometrial carcinogenesis. J Clin Oncol 2006;29: 4783-4791.

6 Horree N, van Diest PJ, van der Groep P, et al. Progressive derailment of cell cycle regulators in endometrial carcinogenesis. J Clin Pathol 2008;61:36-42.

7 Michalides RJAM. Cell cycle regulators: mechanisms and their role in aetiology, prognosis, and treatment of cancer. J Clin Pathol 1999;52:555-568.

8 Sherr CJ. The INK4a/ARF network in tumour suppression. Nat Rev Mol Cell Biol 2001;2:731-737.

9 Kikuchi A. Tumor formation by genetic mutations in the components of the Wnt signalling pathway. Cancer Sci 2003;94:225-229.

10 Sherr CJ, McCormick F. The RB and p53 pathways in cancer. Cancer Cell 2002;2:103-112.

11 Fodde $\mathrm{R}$, Brabletz $\mathrm{T}$. Wnt/ $\beta$-catenin signalling in cancer stemness and malignant behaviour. Curr Opin Cell Biol 2007;19:150-158.

12 Guarino M, Rubino B, Ballabio G. The role of epithelial-mesenchymal transition in cancer pathology. Pathology 2007;39:305-318.

13 Thiery JP. Epithelial-mesenchymal transitions in tumour progression. Nat Rev Cancer 2002;2:442-454.

14 Hugo H, Ackland ML, Blick T, et al. Epithelial-mesenchymal and mesenchymal-epithelial transitions in carcinoma progression. J Cell Physiol 2007;213:374-383.

15 Semczuk A, Cybulski M, Tomaszewski J, et al. Clinicoprognostic significance of $\mathrm{pRb} 1$ pathway alterations in uterine endometrial adenocarcinoma. Cancer Genet Cytogenet 2004;154:186-189.

16 Saegusa M, Hashimura M, Kuwata T, et al. Induction of p16 $6^{\mathrm{INK} 4 \mathrm{~A}}$ mediated by $\beta$-catenin in a TCF4-independent manner: implications for alterations in $\mathrm{p} 16^{\mathrm{INK} 4 \mathrm{~A}}$ and $\mathrm{pRb}$ expression during trans-differentiation of endometrial carcinoma cells. Int J Cancer 2006;119: 2294-2303.

17 Le NH, Franken P, Fodde R. Tumour-stroma interactions in colorectal cancer: converging on $\beta$-catenin activation and cancer stemness. Br J Cancer 2008;98: 1886-1893.

18 Murray SK, Young RH, Scully RE. Unusual epithelial and stromal changes in myoinvasive endometrioid adenocarcinomas: a study of their frequency, associated diagnostic problems, and prognostic significance. Int J Gynecol Pathol 2003;22:324-333.

19 Stewart CJR, Brennan BA, Leung YC, et al. MELF pattern invasion in endometrial carcinoma: association with low-grade, myoinvasive endometrioid tumours, focal mucinous differentiation and vascular invasion. Pathology (in press).

20 Stewart CJR, Little L. Immunophenotypic features of MELF pattern invasion in endometrial adenocarcinoma: evidence for epithelial-mesenchymal transition. Histopathology (in press).

21 Silva EG, Deavers MT, Bodurka DC, et al. Association of low-grade endometrioid carcinoma of the uterus and ovary with undifferentiated carcinoma: a new type of dedifferentiated carcinoma? Int J Gynecol Pathol 2006; 25:52-58.

22 Prall F. Tumour budding in colorectal carcinoma. Histopathology 2007;50:151-162.

23 Brabletz T, Hlubek F, Spaderna S, et al. Invasion and metastases in colorectal cancer: epithelial-mesenchymal 
transition, mesenchymal-epithelial transition, stem cells and $\beta$-catenin. Cells Tissues Organs 2005; 179:56-65.

$24 \mathrm{Wu}$ W, Slomovitz BM, Soliman PT, et al. Correlation of cyclin D1 and cyclin D3 overexpression with the loss of PTEN expression in endometrial carcinoma. Int J Gynecol Cancer 2006;16:1668-1672.

25 Quddus MR, Latkovich P, Castellani WJ, et al. Expression of cyclin D1 in normal, metaplastic, hyperplastic endometrium and endometrial carcinoma suggests a role in endometrial carcinogenesis. Arch Pathol Lab Med 2002;126:459-463.

26 Cao QJ, Einstein MH, Anderson PS, et al. Expression of COX-2, Ki-67, cyclin D1 and P21 in endometrial endometrioid carcinomas. Int J Gynecol Pathol 2002; 21:147-154.

27 Semczuk A, Miturski R, Skomra D, et al. Expression of the cell-cycle regulatory proteins (pRb, cyclin D1, p16 ${ }^{\text {INK4A }}$ and cdk4) in human endometrial cancer: correlation with clinicopathological features. Arch Gynecol Obstet 2004;269:104-110.

28 Nikaido T, Li S-F, Shiozawa T, et al. Coabnormal expression of cyclin D1 and p53 protein in human uterine endometrial carcinomas. Cancer 1996;78: 1248-1253.

29 Ito K, Sasano H, Yoshida Y, et al. Immunohistochemical study of cyclins D and E and cyclin dependent kinase (cdk) 2 and 4 in human endometrial carcinoma. Anticancer Res 1998;18:1661-1664.

30 Horree N, van Diest PJ, Sie-Go DMDS, et al. The invasive front in endometrial carcinoma: higher proliferation and associated derailment of cell cycle regulators. Hum Pathol 2007;38:1232-1238.

31 Kobayashi K, Sagae S, Nishioka Y, et al. Mutations of the beta-catenin gene in endometrial carcinomas. Jpn J Cancer Res 1999;90:55-59.

32 Moreno-Bueno G, Hardisson D, Sarrio D, et al. Abnormalities of $\mathrm{E}$ - and P-cadherin and catenin (beta-, gamma-catenin, and p120ctn) expression in endometrial cancer and endometrial atypical hyperplasia. J Pathol 2003;199:471-478.

33 Saegusa M, Hashimura M, Yoshida T, et al. $\beta$-Catenin mutations and aberrant nuclear expression during endometrial tumorigenesis. Br J Cancer 2001;84: 209-217.

34 Machin P, Catasus L, Pons C, et al. CTNNB1 mutations and beta-catenin expression in endometrial carcinomas. Hum Pathol 2002;33:206-212.

35 Fukuchi T, Sakamoto $\mathrm{M}$, Tsuda $\mathrm{H}$, et al. $\beta$-Catenin mutation in carcinoma of the uterine endometrium. Cancer Res 1998;58:3526-3528.

36 Nei H, Saito T, Yamasaki H, et al. Nuclear localization of beta-catenin in normal and carcinogenic endometrium. Mol Carcinog 1999;25:207-218.

37 Palacios J, Catasus L, Moreno-Bueno G, et al. Beta- and gamma-catenin expression in endometrial carcinoma. Relationship with clinicopathological features and microsatellite instability. Virchows Arch 2001;438: 464-469.

38 Shih H-C, Shiozawa T, Miyamoto T, et al. Immunohistochemical expression of E-cadherin and $\beta$-catenin in the normal and malignant human endometrium: an inverse correlation between E-cadherin and nuclear $\beta$-catenin expression. Anticancer Res 2004;24: 3843-3850.

39 Stefansson IM, Salvesen HB, Akslen LA. Prognostic impact of alterations in P-cadherin expression and related cell adhesion markers in endometrial cancer. J Clin Oncol 2004;22:1242-1252.

40 Gupta GP, Massague J. Cancer metastasis: building a framework. Cell 2006;127:679-695.

41 O’Neill CJ, McCluggage WG. p16 expression in the female genital tract and its value in diagnosis. Adv Anat Pathol 2006;13:8-15.

42 Semczuk A, Boltze C, Marzec B, et al. p16 ${ }^{I N K 4 A}$ alterations are accompanied by aberrant protein immunostaining in endometrial carcinomas. J Cancer Res Clin Oncol 2003;129:589-596.

43 Tsuda H, Yamamoto K, Inoue $\mathrm{T}$, et al. The role of p16-cyclin D/CDK-pRb pathway in the tumorigenesis of endometrioid-type endometrial carcinoma. $\mathrm{Br} \mathrm{J}$ Cancer 2000;82:675-682.

44 Salvesen HB, Das S, Akslen LA. Loss of nuclear p16 protein expression is not associated with promoter methylation but defines a subgroup of aggressive endometrial carcinomas with poor prognosis. Clin Cancer Res 2000;6:153-159.

45 Milde-Langosch K, Riethdorf L, Bamberger A-M, et al. P16/MTS1 and pRB expression in endometrial carcinomas. Virchows Arch 1999;434:23-28.

46 Nakashima R, Fujita M, Enomoto T, et al. Alteration of p16 and p15 genes in human uterine tumours. Br J Cancer 1999;80:458-467.

47 Engelsen IB, Stefansson I, Akslen LA, et al. Pathologic expression of p53 or p16 in preoperative curettage specimens identifies high-risk endometrial carcinomas. Am J Obstet Gynecol 2006;195:979-986.

48 Reid-Nicholson $\mathrm{M}$, Iyengar $\mathrm{P}$, Hummer AJ, et al. Immunophenotypic diversity of endometrial adenocarcinomas: implications for differential diagnosis. Mod Pathol 2006;19:1091-1100.

49 McCluggage WG, Jenkins D. p16 immunoreactivity may assist in the distinction between endometrial and endocervical adenocarcinoma. Int J Gynecol Pathol 2003;22:231-235.

50 Ansari-Lari MA, Staebler A, Zaino RJ, et al. Distinction of endocervical and endometrial adenocarcinomas. Immunohistochemical p16 expression correlated with human papillomavirus (HPV) DNA detection. Am J Surg Pathol 2004;28:160-167.

51 Shiozawa T, Nikaido T, Shimizu M, et al. Immunohistochemical analysis of the expression of cdk4 and p16 $6^{\text {INK4 }}$ in human endometrioid-type endometrial carcinoma. Cancer 1997;80:2250-2256.

52 Jie G, Zhixiang S, Lei S, et al. Relationship between expression and methylation status of $\mathrm{p} 16^{\mathrm{INK} 4 \mathrm{a}}$ and the proliferative activity of different areas' tumour cells in human colorectal cancer. Int J Clin Pract 2007;61: 1523-1529.

53 Jung A, Schrauder M, Oswald U, et al. The invasion front of human colorectal adenocarcinomas shows co-localization of nuclear beta-catenin, cyclin D1, and p16INK4A and is a region of low proliferation. Am J Pathol 2001;159:1613-1617. 